\title{
Noninvasive positive-pressure ventilation for extubation failure after cardiac surgery: Pilot safety evaluation
}

\author{
Luca S. De Santo, MD, ${ }^{\mathrm{a}}$ Ciro Bancone, MD, ${ }^{\mathrm{b}}$ Giuseppe Santarpino, MD, ${ }^{\mathrm{b}}$ Gianpaolo Romano, MD, ${ }^{\mathrm{c}}$ Alessandro Della Corte, MD, \\ Mariano Vicchio, MD, ${ }^{\mathrm{b}}$ Antonio De Pietro, MD, ${ }^{\mathrm{c}}$ Nicola Galdieri, MD, ${ }^{\mathrm{c}}$ and Maurizio Cotrufo, MD, FECTS ${ }^{\mathrm{b}}$
}

\begin{abstract}
Objective: Extubation failure is a serious complication after cardiac surgery. The role of noninvasive positivepressure ventilation for acute respiratory failure in patients undergoing cardiac surgery is unknown. This study aimed to assess the safety of implementing noninvasive positive-pressure ventilation in this setting and its impact on lung function and operative outcomes.
\end{abstract}

\begin{abstract}
Methods: In a 6-month pilot prospective survey, the study population comprised 43 patients (32were male with a mean age of $65.73 \pm 9$ years; 3 heart transplantations, 18 coronary artery bypass grafts, 5 aortic dissections, and 17 valvular procedures; 34 active smokers, 25 with medically treated chronic obstructive pulmonary disease, 21 emergency/urgency procedures) who required noninvasive positive-pressure ventilation for acute respiratory failure after initial weaning from a respirator. The cause of acute respiratory failure (classified as post-cardiopulmonary bypass lung injury in $48.8 \%$ [ 21 patients], cardiogenic edema in $30.2 \%$ [ 13 patients], and pneumonia in $21 \%$ [9 patients]), length of noninvasive positive-pressure ventilation support, respiratory ratios (arterial oxygen tension/fraction of inspired oxygen assessed immediately before noninvasive positive-pressure ventilation, and every 6 hours after institution of pressure ventilation), and need for reintubation along with a set of predefined safety parameters were recorded.
\end{abstract}

Results: The mean length of noninvasive positive-pressure ventilation support was $33.8 \pm 24.04$ hours. Plotting respiratory ratios with length of noninvasive positive-pressure ventilation supports a significant improvement was already evident within the first 6-hour frame $(133.6 \pm 39.5$ vs $205 \pm 65.7 ; P<.001)$ for all causes. Noninvasive positive-pressure ventilation prevented intubation in $74.4 \%$ of the patients, with satisfactory recovery for postcardiopulmonary bypass lung injury and cardiogenic dysfunction $(90.5 \%$ and $69.2 \%$, respectively) and poor results $(55 \%$ reintubated) in those treated for pneumonia. Noninvasive positive-pressure ventilation safety approached $97.7 \%$.

Conclusion: In appropriate candidates, noninvasive positive-pressure ventilation exerts favorable effects on lung function, preventing reintubation. The cost-effectiveness of its systematic use in this setting should be assessed.

Because of increasingly complex surgical candidates, extubation failure after cardiac surgery is becoming a common complication of perioperative course. ${ }^{1-4}$ Reinstitution of tracheal intubation and mechanical ventilation increases the use of intensive care unit and hospital resources and severely affects morbidity and mortality. ${ }^{1,3,5}$ Noninvasive positivepressure ventilation (NPPV) has become a standard of care in acute respiratory failure. ${ }^{6,7}$ However, little and controversial data are available on its usefulness in post-extubation failure after major surgery and in particular after cardiac procedures. ${ }^{8-13}$ Further, outcome descriptors for noninvasive ventilation have been poorly defined.

From the University of Foggia School of Medicine, ${ }^{\text {a }}$ Foggia, Italy; Department of Cardiothoracic and Respiratory Sciences, Second University of Naples, V. Monaldi Hospital, ${ }^{\mathrm{b}}$ Naples, Italy; and Department of Cardiovascular Surgery and Transplantation, V. Monaldi Hospital, ${ }^{\mathrm{c}}$ Naples, Italy.

Received for publication Oct 24, 2007; revisions received Feb 4, 2008; accepted for publication July 5, 2008.

Address for reprints: Luca S. De Santo, MD, Department of Cardiothoracic and Respiratory Sciences, Second University of Naples, V. Monaldi Hospital, Via L. Bianchi, 80131 Naples, Italy (E-mail: lucas.desanto@ospedalemonaldi.it).

J Thorac Cardiovasc Surg 2009;137:342-6

$0022-5223 / \$ 36.00$

Copyright (c) 2009 by The American Association for Thoracic Surgery doi:10.1016/j.jtcvs.2008.07.067
The primary purpose of this 6-month pilot survey was to document the safety of implementing NPPV in this setting and its impact on lung function and operative outcomes. Secondary end points were to investigate the role of the underlying cause of respiratory failure as NPPV outcome predictor.

\section{MATERIALS AND METHODS Patient Population}

Between January and July of 2007, we enrolled 43 consecutive adult patients who underwent cardiac surgery procedures at our tertiary referral center and experienced hypoxemic acute respiratory failure after prior successful weaning from ventilator support and extubation. The study was conducted in accordance with the principles of the Declaration of Helsinki. The local ethics committee approved the protocol, and written informed consent was always obtained.

\section{Extubation Criteria}

According to current guidelines, ${ }^{14}$ after a conventional weaning protocol, patients were extubated when they met the following conditions: awake and cooperative and no evidence of focal or global neurologic deficit; bleeding from mediastinal and pleural drainage less than $100 \mathrm{~mL} / \mathrm{h}$; urine output of greater than $0.5 \mathrm{~mL} / \mathrm{kg} / \mathrm{h}$; hemodynamic stability without the need for high dosage of vasopressors, inotropes, or intraaortic balloon pump; no severe arrhythmia; respiratory rate less than 30 breaths/min, arterial oxygen 


\section{Abbreviations and Acronyms \\ $\mathrm{CPB}=$ cardiopulmonary bypass \\ $\mathrm{FIO}_{2}=$ fraction of inspired oxygen \\ $\mathrm{NPPV}=$ noninvasive positive-pressure ventilation \\ $\mathrm{PaO}_{2}=$ arterial oxygen tension}

tension $\left(\mathrm{PaO}_{2}\right)$ greater than $70 \mathrm{~mm} \mathrm{Hg}$, with fraction of inspired oxygen $\left(\mathrm{FIO}_{2}\right)$ less than 0.35 and positive end-expiratory pressure of $5 \mathrm{~cm} \mathrm{H}_{2} \mathrm{O}$ or less; normocapnia, with pressure support of $10 \mathrm{~cm} \mathrm{H}_{2} \mathrm{O}$ or less; and body core temperature greater than $36.6^{\circ} \mathrm{C}$, absence of persistent or worsening metabolic acidosis with arterial $\mathrm{pH}$ greater than 7.35, and bicarbonate of greater than $20 \mathrm{mmol} / \mathrm{L}$. Prophylactic respiratory physiotherapy was always performed through intense physical therapy, incentive spirometry, and early mobilization. ${ }^{15}$

\section{Noninvasive Ventilation}

As recommended by current guidelines, ${ }^{6}$ NPPV was applied when acute respiratory distress occurred, including severe dyspnea at rest and all of the following: respiratory rate more than 30 breaths/min; hypoxemia arterial partial pressure of oxygen/inspired oxygen fraction ratio $\left(\mathrm{PaO}_{2} / \mathrm{FiO}_{2}\right)$ less than 200 while breathing oxygen through a Venturi mask; and active contraction of the accessory muscles of respiration or paradoxical abdominal motion after ensuring adequate analgesia, ability to protect airway, and airway secretion clearance. NPPV was achieved using a flow generator with an adjustable inspiratory oxygen fraction set to deliver a flow up to $140 \mathrm{~L} /$ min and a spring-loaded expiratory pressure valve and applied using a latex-free polyvinyl chloride transparent helmet. ${ }^{16}$ For all patients, NPPV was started at $7.5 \mathrm{~cm} \mathrm{H}_{2} \mathrm{O}$. The level could be decreased to $5 \mathrm{~cm} \mathrm{H}_{2} \mathrm{O}$ or increased to $10 \mathrm{~cm} \mathrm{H}_{2} \mathrm{O}$ as needed according to clinical response and tolerance. After the first hour, $\mathrm{FiO}_{2}$ was adjusted to keep a $\mathrm{PaO}_{2}$ greater than 70 $\mathrm{mm} \mathrm{Hg}$. NPPV was delivered until endotracheal intubation, death, or fulfillment of the following cessation criteria: $\mathrm{PaO}_{2} / \mathrm{FIO}_{2}$ greater than 300 , $95 \%$ less than arterial oxygen saturation $\left(\mathrm{SaO}_{2}\right)$ less than $100 \%$, under $\mathrm{FIO}_{2}$ equal or lower than of $40 \%$ or less without NPPV, and respiratory rate less than 30 breaths/min. Occurrence of $\mathrm{CO}_{2}$ rebreathing was avoided by delivering high fresh gas flow (never $<30 \mathrm{~L} / \mathrm{min}$ ) to wash exhaled $\mathrm{CO}_{2}$ away from the closed volume inside the helmet.

Patients had continuous electrocardiographic and oxygen saturation monitoring along with invasive hemodynamic monitoring. Arterial blood gas levels were determined at baseline and at 1 and 6 hours thereafter.

\section{Exclusion Criteria}

Patients with any of the following were not included in the trial: lack of consciousness; deformity of upper airways; acute bleeding of the upper airways; severe pulmonary emphysema with bullae; hypercapnic respiratory failure, severe sepsis; hypoxemic respiratory failure after accidental self- extubation; or pneumothorax.

\section{Criteria for Endotracheal Intubation}

Patients who failed to improve with noninvasive ventilation underwent endotracheal intubation with cuffed endotracheal tubes and were mechanically ventilated. Reintubation was performed if 1 major or 2 minor criteria were present. Major criteria were respiratory arrest, loss of consciousness, severe agitation, hemodynamic instability, and cardiogenic shock. Minor criteria were respiratory rate more than 35 breaths/min and higher than admission value, arterial $\mathrm{pH}$ less than 7.30 and lower than admission value, $\mathrm{PaO}_{2}$ less than $45 \mathrm{~mm} \mathrm{Hg}$ despite oxygen supplementation, neurologic deterioration, and weak cough reflex with secretion accumulation.

\section{End Points and Definitions}

The primary outcome variables were the need for endotracheal intubation, $\mathrm{PaO}_{2} / \mathrm{FIO}_{2}$ ratios, and hospital morbidity and mortality of NPPV. The secondary end point was to evaluate the role of the underlying cause of respiratory failure as a predictor on noninvasive ventilation failure. Acute respiratory failure was classified as follows: post-cardiopulmonary bypass (CPB) lung impairment (acute lung injury criteria without cardiogenic compromise and negative "pneumonia" criteria); pneumonia (positive microbiology culture and appropriate imaging and clinical features, including a temperature of $>38.5^{\circ} \mathrm{C}$ or $\left\langle 35^{\circ} \mathrm{C}\right.$ and white blood cell count $>11 \times$ $10^{9}$ or $<4 \times 10^{9}$ cell/L); and cardiogenic edema (pulmonary capillary wedge pressure $>18 \mathrm{~mm} \mathrm{Hg}$, postoperative cardiac index $(\mathrm{CI})<1.8 \mathrm{~L} /$ $\mathrm{min} / \mathrm{m}^{2}$ and high-dosage catecholamines, intraaortic balloon pump, postoperative myocardial infarction, or severe arrhythmia).

Early improvement in gas exchange was defined as the ability to increase $\mathrm{PaO}_{2} / \mathrm{FIO}_{2}$ more than 200 or an increase in this ratio of more than 100 from baseline. Delayed improvement in gas exchange was defined as the ability to maintain the early improvement in respiratory ratios until ventilation was discontinued, as confirmed by serial blood gas measurements. Safety parameters were as follows: skin necrosis, conjunctivitis, sinusitis, gastric distension, pneumothorax, nosocomial pneumonia, stress gastrointestinal tract ulcer and bleeding, aspiration, cardiac arrest, and claustrophobia.

Perioperative patient variables were collected according to the standards of the Society of Thoracic Surgeons by 3 full-time research assistants and maintained in a computer database format.

\section{Data Analysis}

The Statistical Package for the Social Sciences software (version 10.1; SPSS Inc, Chicago, Ill) was used for statistical analysis. Data were expressed as mean \pm standard deviation, or counts and percentages when appropriate. Differences in categoric variables were compared by means of the chi-square Pearson's test or Fisher's exact test. Continuous variables were analyzed with the 2-tailed Student $t$ test. Predictive factors for NPPV failure were assessed in univariate and multivariable analyses on 21 variables (age, sex, diabetes, active smoking, chronic obstructive pulmonary disease, low left ventricular ejection fraction $[\leq 35 \%]$, operative procedure, operative status, preoperative $\mathrm{PaO}_{2} / \mathrm{FIO}_{2}$, redo operation, $\mathrm{CPB}$ and aortic crossclamp times, need and number of transfusions, surgical reexploration for bleeding, postoperative low cardiac output, postoperative renal insufficiency, length of first intubation, interval between extubation and NPPV, indication for NPPV treatment, and $\mathrm{PaO}_{2} / \mathrm{FiO}_{2}$ ratio $<200$ after first treatment hour). The effect of the underlying cause of respiratory failure on NPPV outcomes was evaluated by exact logistic regression model using LogXact software to account for the small sample size (LogXact-Turbo; CYTEL Software Corporation, Cambridge, Mass).

\section{RESULTS}

Of 465 consecutive patients who underwent operation during the 6-month time frame of this study, post-extubation failure developed in $44(9.46 \%)$. Forty-three patients met study entry criteria and constituted the NPPV group. One patient with post-extubation hypercapnic respiratory failure and severe pulmonary emphysema with bullae was included in the control sample. Baseline characteristics and operative variables of study groups are summarized in Tables 1 and 2 . Patients experiencing post-extubation respiratory failure had a significantly worse preoperative clinical status, underwent more complex surgical procedures, and had a more demanding postoperative course. The length of initial mechanical ventilation was significantly longer in the NPPV group. 
TABLE 1. Perioperative features of study population

\begin{tabular}{lccc}
\hline & Control n $=\mathbf{4 2 2}$ & NPPV n $=\mathbf{4 3}$ & $\boldsymbol{P}$ \\
\hline Age & $62.4 \pm 12.4$ & $65.7 \pm 9.04$ & .039 \\
Sex (female) & $132(31.4 \%)$ & $11(25.6 \%)$ & NS \\
Diabetes & $89(21.1 \%)$ & $11(25.6 \%)$ & NS \\
Smoking & $215(51 \%)$ & $34(79.1 \%)$ & .01 \\
COPD & $21(5) \%$ & $25(58.1 \%)$ & .002 \\
Low LVEF & $44(10.4) \%$ & $17(39.5 \%)$ & .009 \\
Diagnosis: & & & .02 \\
$\quad$ Ischemic & $201(47.6 \%)$ & $26(60.5 \%)$ & \\
$\quad$ Valvular & $164(38.86 \%)$ & $3(7 \%)$ & \\
$\quad$ Thoracic aortic & $44(10.42 \%)$ & $9(20.9 \%)$ & \\
$\quad$ Transplantation & $12(2.84 \%)$ & $3(7 \%)$ & \\
$\quad$ Other & $1(0.24 \%)$ & $2(4.6 \%)$ & \\
Status & & & .01 \\
$\quad$ Elective & $295(69.9 \%)$ & $22(51.2 \%)$ & \\
$\quad$ Urgent & $54(12.8 \%)$ & $12(27.9 \%)$ & \\
Emergency & $73(17.3 \%)$ & $9(20.9 \%)$ & \\
\hline
\end{tabular}

$N P P V$, Noninvasive positive-pressure ventilation; $C O P D$, chronic obstructive pulmonary disease; $L V E F$, left ventricular ejection fraction; $N S$, not significant.

The mean interval between extubation and development of respiratory failure was $30.6 \pm 24.56$ hours. The indications for NPPV treatment were post-CPB lung injury in $48.8 \%$ (21 patients), cardiogenic edema in $30.2 \%$ (13 patients), and pneumonia in $21 \%$ (9 patients).

\section{Clinical Outcomes}

The mean length of NPPV treatment was $33.8 \pm 24.04$ hours. Figure 1 reports the pattern of respiratory ratios during NPPV treatment. Noninvasive ventilation exerted a significant improvement on oxygenation already evident after 1 hour of treatment. Compared with baseline, NPPV treatment was also associated with a significant reduction in respiratory rate (34 [20-60] vs 28 [12-40], $P<.001)$, a significant increase in arterial $\mathrm{pH}$ levels $(7.39$ [7.22-7.58] vs 7.42 [7.3$7.57], P=.01)$, and a decrease in heart rate (104 [40-150] vs 98 [60-145], $P=.003$ ). Treatment compliance was $100 \%$. The rate of adverse events was as low as $2.3 \%$ ( 1 case of gastric distension). Endotracheal reintubation was required in $25.6 \%$ (11 patients). Refractory hypoxemia (36.4\%) with or without respiratory acidosis, agitation/exhaustion $(54.6 \%)$, and hemodynamic instability $(9 \%)$ accounted for endotracheal intubation. The rate of endotracheal intubation was significantly higher in patients with underlying pneumonia compared with those treated for cardiogenic or $\mathrm{CPB}$ lung injury (55\% vs $30.8 \%$ vs $9.5 \%$, respectively, $P=$ .03). The outcomes of patients experiencing extubation failure were poorer than those of the overall control sample.

A subsequent tracheostomy was required in $9.3 \%$ (4 patients) in the NPPV group versus $0.94 \%$ (4 patients) in the control group $(P<.001)$. The lengths of intensive care unit and hospital stay were $18.2 \pm 22.1$ days and 23.6 \pm 32 days in the NPPV group versus $2.4 \pm 4.6$ and 10.9 \pm 9.7 in the control group $(P<.001)$, respectively. Hospital
TABLE 2. Perioperative features of study population

\begin{tabular}{lccc}
\hline & Control $\mathbf{n}=\mathbf{4 2 2}$ & NPPV n $=43$ & $\boldsymbol{P}$ \\
\hline Preoperative $\mathrm{PaO}_{2} / \mathrm{FIO}_{2}$ & $381 \pm 41$ & $357 \pm 60.4$ & .02 \\
Redo & $34(8.04 \%)$ & $5(11.6 \%)$ & .08 \\
Total CPB & $104.8 \pm 58.3$ & $125.13 \pm 52.7$ & .001 \\
Total aortic crossclamp & $66.02 \pm 29.1$ & $67.1 \pm 35.7$ & .01 \\
Blood products & $2.6 \pm 4.2$ & $5.6 \pm 13.4$ & $<.0001$ \\
Surgical reexploration & $4(0.94 \%)$ & $2(4.6 \%)$ & $<.0001$ \\
IABP & $17(4.02 \%)$ & $9(20.9 \%)$ & .0004 \\
CVVH & $19(4.5 \%)$ & $10(23.3 \%)$ & .0004 \\
Invasive monitoring & $19(4.5 \%)$ & $43(100 \%)$ & $<.0001$ \\
Initial MV & $19.1 \pm 26.4$ & $30.7 \pm 31.8$ & $<.0001$ \\
\hline
\end{tabular}

$\mathrm{NPPV}$, Noninvasive positive-pressure ventilation; $\mathrm{PaO}_{2} / \mathrm{FIO}_{2}$, arterial oxygen tension/ fraction of inspired oxygen; $C P B$, cardiopulmonary bypass; $I A B P$, intraaortic balloon pump; $C V V H$, continuous venovenous hemo filtration; $M V$, length of mechanical ventilation (in hours).

mortality was $14 \%$ (6 patients) in the NPPV group versus $6.7 \%$ (28 patients) in the control group $(P<.001)$. Causes of death in the NPPV group were low cardiac output in 3 cases, multiple organ failure in 2 cases, and septic shock in 1 case. Causes of death in controls were low cardiac output in 16 cases, multiple organ failure in 5 cases, and septic shock in 7 cases.

\section{Outcome Predictors}

Perioperative patient characteristics were evaluated as predictors of NPPV failure. Significant univariate predictors were $\mathrm{PaO}_{2} / \mathrm{FIO}_{2}$ less than 200 after the first treatment hour $(P=.005)$, duration of initial intubation $(P=.01)$, and underlying respiratory failure cause $(P<.001)$. Multivariate analysis revealed pneumonia (odds ratio 1.25 ; $95 \%$ confidence interval, $1.01-6.4 ; P<.001)$ as the only independent predictor of unsuccessful noninvasive ventilatory treatment.

\section{DISCUSSION}

This pilot prospective safety evaluation validated the implementation of early NPPV in the setting of post-extubation respiratory failure after cardiac surgery. Indeed, it relieved hypoxemia and helped avoid reintubation in $75 \%$ of patients who already met standard intubation criteria at study entry. Treatment compliance was $100 \%$ with an incidence of adverse events of $2.3 \%$. The use of a helmet as NPPV interface could explain these favorable outcomes compared with published data. ${ }^{16}$ Cause of respiratory failure emerged as the strongest predictor of NPPV failure in agreement with major series. ${ }^{17,18}$ Outcome predictors are important to identify patients who are less likely to improve with noninvasive ventilation, thus requiring closer observation and readily available means of intubation. This is particularly relevant for the patients with severe hypoxemia, in whom unnecessary delays in intubation may imply serious consequences.

The outcomes of NPPV treatment for post-CPB lung impairment were satisfactory in the present series. Such results 


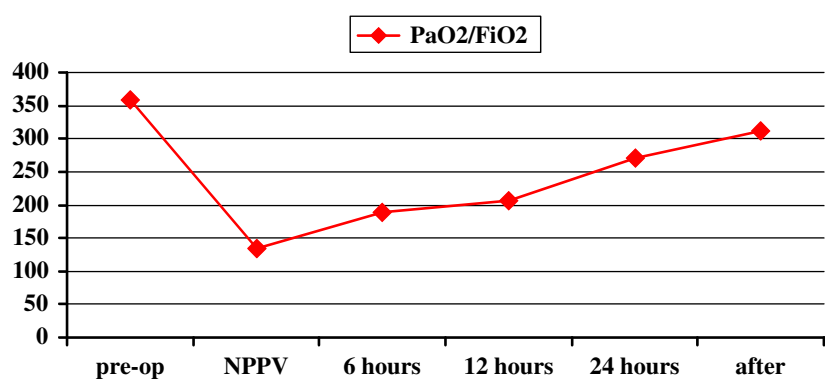

FIGURE 1. Temporal trend of $\mathrm{PaO}_{2} / \mathrm{FIO}_{2}$. NPPV, Noninvasive positivepressure ventilation; $\mathrm{PaO}_{2} / \mathrm{FIO}_{2}$, arterial oxygen tension/fraction of inspired oxygen.

compare favorably with published series and may be explained by the physiologic effects of continuous positive airway pressure and partly by patient stratification in this strictly selective study. ${ }^{19,20}$

Published research has suggested that continuous positive airway pressure and NPPV impart hemodynamic benefit in patients with heart failure by increasing intrathoracic pressure, which, in turn, reduces both cardiac preload (by impeding cardiac filling) and cardiac afterload (by reducing left ventricular transmural pressure). ${ }^{21,22}$ High-level evidence supports the use of continuous positive airway pressure in treating cardiogenic pulmonary edema. Pang and colleagues $^{23}$ reported that, compared with standard therapy, continuous positive airway pressure was associated with less need for intubation (risk difference $26 \%$; $95 \%$ confidence interval, 13-38) and that there was a trend toward lower hospital mortality (risk difference $6.6 \%$; $95 \%$ confidence interval, 3-16). NPPV treatment for patients with cardiogenic pulmonary edema also proved effective in this study. Intubation rate averaged a satisfactory $30.8 \%$. Concomitant close hemodynamic monitoring, aggressive evidence-based inodilator support, extensive use of intraaortic balloon pump, and renal replacement therapy may justify these results. Nevertheless, lower intubation rates have been reported in published series. ${ }^{7,17,23}$ These may be explained by a different case mix and in particular by the significant extent of preoperative cardiac impairment, the complex comorbidity profiles, and the exclusive post-major surgical procedure setting of present series.

The NPPV failure rate proved significantly higher in patients affected by pneumonia. In close accordance with major series, ${ }^{18}$ strong evidence is emerging that once satisfactory oxygenation and ventilatory assistance are attained by NPPV, the major determinant of the outcome is the response to medical therapy and the resolution of the underlying disease. Pneumonia has a relatively slow onset, and time is required for conventional medical therapy to show its effects. Conventional endotracheal intubation still seems to be the best intervention in this patient subset. Despite successful NPPV treatment, mortality rates in this series are as high as $14 \%$, and significantly higher than those observed in controls. Such results fit with the lower limits of reported fatality rates and may be explained by the complex case mix and development of multiorgan involvement. ${ }^{1-5}$

\section{Study Limitations}

Management of post-extubation failure is a highly controversial issue, with a limited number of prospective randomized analyses partially biased by limited samples of unselected patient populations. Despite appropriate data analysis, the limited study sample in the present series inevitably hampers the inherent statistical power. Nevertheless, the prospective setting, the uniformity of perioperative management standards inherent to a single-center analysis, and, above all, the strict patient selection still support a definite clinical relevance. Given the inherent limits of both endotracheal reintubation and simple oxygen supplementation, this feasibility evaluation of NPPV, added to standard intensive care treatment, was not conducted in a randomized fashion. Stringent exclusion and intubation criteria, drawn from evidence-based guidelines, guaranteed safe and effective "state of the art" patient care.

\section{CONCLUSIONS}

The present safety study shows that NPPV is a safe adjunct in the treatment of post-extubation failure in patients undergoing cardiac surgery. Together with intensive standard medical management, strict patient selection is crucial for NPPV success. Patients affected by pneumonia seem to benefit least from this ventilatory modality. Further studies are needed to address the proper timing and selection criteria and to validate the cost-effectiveness of the routine implementation of this strategy.

The authors thank Leone Roberto, student at the School of Medicine of the Second University of Naples, for cooperation in data mining and storage.

\section{References}

1. Rady MY, Ryan T. Perioperative predictors of extubation failure and the effect on clinical outcome after cardiac surgery. Crit Care Med. 1999;27:340-7.

2. Cohn WE, Sellke FW, Sirois C, Lisbon A, Johnson RG. Surgical ICU recidivism after cardiac operations. Chest. 1999;116:688-92.

3. Bardell T, Legare JF, Buth KJ, Hirsch GM, Ali IS. ICU readmission after cardiac surgery. Eur J Cardiothorac Surg. 2003;23:354-9.

4. Vohra HA, Goldsmith IR, Rosin MD, Briffa NP, Patel RL. The predictors and outcome of recidivism in cardiac ICUs. Eur J Cardiothorac Surg. 2005;27:508-11.

5. Hein OV, Birnbaum J, Wernecke KD, Konertz W, Jain U, Spies C. Three-year survival after four major post-cardiac operative complications. Crit Care Med. 2006;34:2729-37.

6. Evans TW. International Consensus Conferences in Intensive Care Medicine: non-invasive positive pressure ventilation in acute respiratory failure. Organised jointly by the American Thoracic Society, the European Respiratory Society, the European Society of Intensive Care Medicine, and the Societe de Reanimation de Langue Francaise, and approved by the ATS Board of Directors, December 2000 Intensive Care Med. 2001;27:166-78.

7. Hess DR. The evidence for noninvasive positive-pressure ventilation in the care of patients in acute respiratory failure: a systematic review of the literature. Respir Care. 2004;49:810-29. 
8. Keenan SP, Sinuff T, Cook DJ, Hill NS. Does noninvasive positive pressure ventilation improve outcome in acute hypoxemic respiratory failure? A systematic review. Crit Care Med. 2004;32:2516-23.

9. Kindgen-Milles D, Buhl R, Gabriel A, Böhner H, Müller E. Nasal continuous positive airway pressure: a method to avoid endotracheal reintubation in postoperative high-risk patients with severe nonhypercapnic oxygenation failure. Chest. 2000;117:1106-11.

10. Esteban A, Frutos-Vivar F, Ferguson ND, Arabi Y, Apezteguía C, González M, et al. Noninvasive positive-pressure ventilation for respiratory failure after extubation. N Engl J Med. 2004;350:2452-60.

11. Agarwal R, Aggarwal AN, Gupta D, Jindal SK. Role of noninvasive positivepressure ventilation in postextubation respiratory failure: a meta-analysis. Respir Care. 2007;52:1472-9.

12. Chen XF, Ye JL. Efficacy and safety of non-invasive positive pressure ventilation in the care of dyspnea after cardiac surgery. Zhongguo Wei Zhong Bing Ji Jiu Yi Хue. 2007; 19:542-5.

13. Pasquina $\mathrm{P}$, Merlani P, Granier JM, Ricou B. Continuous positive airway pressure versus noninvasive pressure support ventilation to treat atelectasis after cardiac surgery. Anesth Analg. 2004;99:1001-8.

14. MacIntyre NR. Evidence-based ventilator weaning and discontinuation. Respir Care. 2004;49:830-6.

15. Pasquina $P$, Tramèr MR, Walder $B$. Prophylactic respiratory physiotherapy after cardiac surgery: systematic review. BMJ. 2003;327:1379-85.
16. Antonelli M, Conti G, Pelosi P, Gregoretti C, Pennisi MA, Costa R, et al. New treatment of acute hypoxemic respiratory failure: noninvasive pressure support ventilation delivered by helmet: a pilot controlled trial. Crit Care Med. 2002;30:602-8.

17. Nava S, Ceriana P. Causes of failure of non-invasive mechanical ventilation. Respir Care. 2004;49:295-303.

18. Hess DR. Noninvasive positive-pressure ventilation and ventilator-associated pneumonia. Respir Care. 2005;50:924-9.

19. Pasquina P, Merlani P, Granier JM, Ricou B. Continuous positive airway pressure versus noninvasive pressure support ventilation to treat atelectasis after cardiac surgery. Anesth Analg. 2004;99:1001-8.

20. Asimakopoulos G, Smith PL, Ratnatunga CP, Taylor KM. Lung injury and acute respiratory distress syndrome after cardiopulmonary bypass. Ann Thorac Surg. 1999;68:1107-15.

21. Hoffmann B, Jepsen M, Hachenberg T, Huth C, Welte T. Cardiopulmonary effects of non-invasive positive pressure ventilation (NPPV) - a controlled, prospective study. Thorac Cardiovasc Surg. 2003;51:142-6.

22. Shekerdemian LS, Bush A, Lincoln C, Shore DF, Petros AJ, Redington AN. Cardiopulmonary interactions in healthy children and children after simple cardiac surgery: the effects of positive and negative pressure ventilation. Heart. 1997; 78:587-93.

23. Pang D, Keenan SP, Cook DJ, Sibbald WJ. The effect of positive pressure airway support on mortality and the need for intubation in cardiogenic pulmonary edema: a systematic review. Chest. 1998;114:1185-92. 